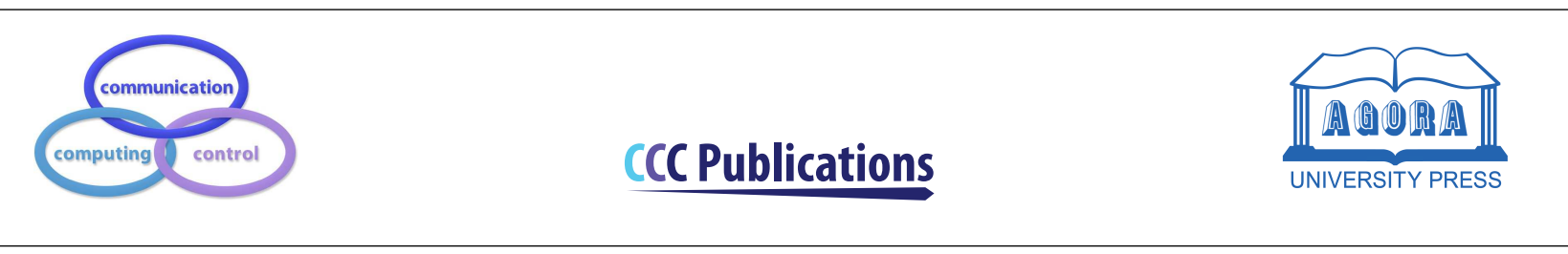

\title{
A Multi-criteria Picture Fuzzy Decision-making Model for Green Supplier Selection based on Fractional Programming
}

\author{
G. Cao
}

\section{Guo Cao*}

1. School of Management

Northwestern Polytechnical University

127 West Youyi Road, Beilin District, Xi'an Shaanxi, 710072, P.R.China

2. School of Economics and Management, Changzhou Institute of Technology

666 Liahoe Road, Changzhou, Jiangsu 213032, China

*Corresponding author: caog@czu.cn

\begin{abstract}
Due to the increasing complexity in green supplier selection, there would be some important issues for expressing inherent uncertainty or imprecision of decision makers' cognitive information in decision making process. As an extension of intuitionistic fuzzy sets (IFSs) and neutrosophic sets (NSs), picture fuzzy sets (PFSs) can better model and represent the hesitancy and uncertainty of decision makers' preference information. In this study, an attempt has been made to present a multi-criteria picture fuzzy decision-making model for green supplier selection based on fractional programming. In this approach, the ratings of alternatives and weights of criteria are represented by PFSs and IFSs, respectively. Based on the available information, some pairs of fractional programming models are derived from the Technique for Order Preference by Similarity to an Ideal Solution (TOPSIS) and the proposed biparametric picture fuzzy distance measure to determine the relative closeness coefficient intervals of green suppliers, which are aggregated for the criteria to generate the ranking order of all green suppliers by computing their optimal degrees of membership based on the ranking method of interval numbers. Finally, an example is conducted to validate the effectiveness of the proposed multi-criteria decision making (MCMD) method.

Keywords: multi-criteria decision making (MCMD), picture fuzzy sets (PFSs), fractional programming, biparametric picture fuzzy distance measure, green supplier selection.
\end{abstract}

\section{Introduction}

In the past two decades, increased environmental pollution is forcing companies to obtain sensitivity to environmental awareness [6]. Manufacturers have applied various approaches to improve their supply chain's environment performance [25]. Green supply chain integrates environmental thinking into supply chain management, including product design, material procurement, production processes, product delivery [2], and managing end-of-life products and waste. Green supplier selection is a critical component of the green supply chain management strategy [10, 23]. As a result, many researchers 
and practitioners have put their efforts on developing various strategies to improve the green supply chain management process.

It has been asserted that selecting the right green suppliers has proven to be one of the key issues faced by operations and purchasing managers to remain competitive [15], and also helps manufacturers in increasing customer satisfaction and quality of the product. Since it requires considering many new problems, it is obvious that the decision-making process would be more complex and comprehensive in the green supply chain management. An important phenomenon among these issues is uncertain and imprecise data. If the fuzziness and vagueness presented in the decision-making process is ignored, the results could be misleading. Aiming at reflecting the bounded rationality of decision makers, Fuzzy set theory is of importance as a result of its merits of mathematically expressing ambiguity and vagueness. Numerous decision making methods has been extended to multiple fuzzy environments, such as Neutrosophic sets, Pythagorean fuzzy sets [9], Hesitant fuzzy sets, Intuitionistic fuzzy sets, and Probability hesitant fuzzy sets [16]. Based on the concept of fuzzy sets (FSs), Atanassov [3] first introduced the concept of IFS, which is an extension of Zadeh's FSs [8]. And then, Cuong [7] proposed the concept of the picture fuzzy set (PFS) to address such a problem that human opinions involve several types of answers such as yes, abstain, no, or refusal and so on. PFS is characterized by a positive-membership function $(\mu(x))$, a negative-membership function $(\nu(x))$ and a neutralmembership function $(\gamma(x))$ such that $0 \leq \mu(x)+\nu(x)+\gamma(x) \leq 1$. Different from NS, there is a restriction on the sum of the three types of membership degrees in PFS, which implies that they are dependent with each other. PFS is also a very effective tool to express inherent uncertainty or imprecision of decision makers' cognitive information in decision making process. However, the application of multi-criteria picture fuzzy decision-making for green supplier selection is a limited area of study in literature.

Green supplier selection is a multi-criteria decision-making problem [14]. Decisions are performed based on the assessment of quantitative and qualitative criteria in two fields including economic and environmental criteria. Nowadays, various techniques were adopted to select green suppliers. Liang et al. presented a multi-criteria decision making method based on multi-granularity interval 2-Tuple fuzzy linguistic set for solving the green supplier selection problems. Govindan et al. [14] proposed an approach integrated of multi-criteria decision making and multi-objective linear programming. Lo et al. [20] developed a hybrid model that integrated the best-worst method, TOPSIS and fuzzy multi-objective linear programming to solve problems in green supplier selection and order allocation. Gitinavard et al. proposed an extended elimination and choice translating reality method in intervalvalued hesitant fuzzy environment [13]. Mohammadi et al. presented a method of supplier selection based on interval type-2 fuzzy sets to deal with uncertain information. It can be concluded that various studies have focused on different fuzzy-based techniques to assess and select green suppliers, including probabilistic linguistic preference relations[11], interval type-2 fuzzy sets[18, 22, 29], intuitionistic uncertain linguistic set[17], triangular fuzzy number[4], intuitionistic fuzzy sets[21, 32], trapezoidal fuzzy numbers [28? ] and neutrosophic set [1]. Most of these studies are based on the assumptions that the weights of a criterion are represented by the known crisp numbers. However, there exist little investigation on multi-attribute decision-making problems with both ratings of green suppliers on criteria and weights being expressed with fuzzy sets [12]. Thus, an interesting and important issue is how to utilize the unknown weights information of a criterion to select the most desirable green supplier. Consequently, it is beneficial to combine the advantages of picture fuzzy sets in representing information and the superiority of fractional programming.

In this paper, we propose a new MCDM method with the concept of the relative-closeness coefficient, where the weight and the evaluating value of a criterion are represented by intuitionistic fuzzy sets and picture fuzzy sets, respectively. Moreover, the fractional programming model is used to obtain the optimal weights of the criteria based on TOPSIS and the proposed biparametric picture distance measure, which provides us with a very useful way for dealing with MCDM problems in picture fuzzy environments.

The remainder of this paper is organized as follows. Section 2 introduces the definition of a PFS, some existing picture fuzzy distance measures and the concept of likelihood for interval numbers. Section 3 proposes a new biparametric picture fuzzy distance measure. Based on the concept of 
relative-closeness coefficients, Section 4 establishes some auxiliary fractional programming models and develops a new approach to solve the decision making problems under the PFSs environment. Section 5 presents a numerical example to illustrate the effectiveness of the proposed approach. Finally, a concrete conclusion is drawn in Section 6.

\section{Preliminaries}

In this section, we present some basic definitions and results for PFS, picture fuzzy distance measure and interval numbers.

Definition 1. [7]. A picture fuzzy set (PFS) $A$ in a finite set $X$ is defined as follows:

$\left.A=\left\{<x, \mu_{A}(x), \nu_{A}(x), \gamma_{A}(x)\right\rangle, x \in X\right\}$,

where $\mu_{A}(x), \nu_{A}(x)$ and $\gamma_{A}(x)$ represent the positive-membership function, negative-membership function and neutral-membership function of $x$ to set $A$, respectively. For each point $x$ in $X$, we have $\mu_{A}(x), \nu_{A}(x), \gamma_{A}(x) \rightarrow[0,1]$ and $0 \leq \mu_{A}(x)+\nu_{A}(x)+\gamma_{A}(x) \leq 1$.

Similar to the IFS, $\pi_{A}(x)=1-\left(\mu_{A}(x)+\nu_{A}(x)+\gamma_{A}(x)\right)$ could be called the refusal-membership agree of $x$ to set $A$. For convenience, we can use $x=\left(\mu_{A}, \nu_{A}, \gamma_{A}\right)$ to represent an element in PFSs.

Definition 2. [7]: Given any two PFSs $A$ and $B$ in a finite set $X$, their inclusion, union, intersection and complement are respectively defined as follows:

(1) $A \subseteq B$ iff $\forall x \in X, \mu_{A}(x) \leq \mu_{B}(x), \nu_{A}(x) \geq \nu_{B}(x), \gamma_{A}(x) \geq \gamma_{B}(x)$

(2) $A=B$ iff $\forall x \in X, A \subseteq B$ and $A \supseteq B$

(3) $A \cup B=\left\{x,\left(\max \left(\mu_{A}(x), \mu_{B}(x)\right), \min \left(\nu_{A}(x), \nu_{B}(x)\right), \min \left(\gamma_{A}(x), \gamma_{B}(x)\right)\right\}\right.$

(4) $A \cap B=\left\{x,\left(\min \left(\mu_{A}(x), \mu_{B}(x)\right), \max \left(\nu_{A}(x), \nu_{B}(x)\right), \min \left(\gamma_{A}(x), \gamma_{B}(x)\right)\right\}\right.$

(5) $\operatorname{co} A=\bar{A}=\left\{<x, \nu_{A}(x), \mu_{A}(x), \gamma_{A}(x)>, x \in X\right\}$

Definition 3. $d(A, B)$ is a distance measure between $A \in P F S(X)$ and $B \in P F S(X)$ if it satisfies the following properties:

(1) $0 \leq d(A, B) \leq 1$

(2) if $A=B$, then $d(A, B)=0$

(3) $d(A, B)=d(B, A)$

(4) if $A \subseteq B \subseteq C$, then $d(A, B)+d(B, C) \geq d(A, C)$

Definition 4. [26, 27]. Given any two PFSs $A$ and B, the normalized picture Hamming distance, the normalized picture Euclidean distance and the generalized picture distance measure between $A$ and $B$ are respectively defined as follows:

$$
\begin{aligned}
d_{H}(A, B) & =\frac{1}{n} \sum_{j=1}^{n}\left(\left|\mu_{A}\left(x_{j}\right)-\mu_{B}\left(x_{j}\right)\right|+\left|\nu_{A}\left(x_{j}\right)-\nu_{B}\left(x_{j}\right)\right|+\left|\gamma_{A}\left(x_{j}\right)-\gamma_{B}\left(x_{j}\right)\right|\right) \\
d_{E}(A, B)= & \sqrt{\frac{1}{n} \sum_{j=1}^{n}}\left(\mu_{A}\left(x_{j}\right)-\mu_{B}\left(x_{j}\right)\right)^{2}+\left(\nu_{A}\left(x_{j}\right)-\nu_{B}\left(x_{j}\right)\right)^{2}+\left(\gamma_{A}\left(x_{j}\right)-\gamma\left(x_{j}\right)\right)^{2} \\
d_{G}(A, B) & =\frac{1}{5}\left(\left|\mu_{A}\left(x_{j}\right)-\mu_{B}\left(x_{j}\right)\right|^{P}+\left|\nu_{A}\left(x_{j}\right)-\nu_{B}\left(x_{j}\right)\right|^{P}+\left|\gamma_{A}\left(x_{j}\right)-\gamma\left(x_{j}\right)\right|^{P}\right. \\
& +\left|\max \left(\mu_{A}\left(x_{j}\right), \nu_{B}\left(x_{j}\right)\right)-\max \left(\mu_{B}\left(x_{j}\right), \nu_{A}\left(x_{j}\right)\right)\right|^{P} \\
& \left.+\left|\max \left(\mu_{A}\left(x_{j}\right), \gamma_{B}\left(x_{j}\right)\right)-\max \left(\gamma_{A}\left(x_{j}\right), \mu_{B}\left(x_{j}\right)\right)\right|^{P}\right)^{1 / P}
\end{aligned}
$$


Definition 5. [31]. Given any two interval numbers $a=\left[a^{-}, a^{+}\right]$and $b=\left[b^{-}, b^{+}\right]$, the likelihood of $a \geq b$ is defined as follows:

$$
p\left(a \geq b=\max \left\{\left(1-\max \left(\frac{b^{+}-a^{-}}{L(a)+L(b)}\right), 0\right), 0\right\}\right.
$$

where $L(a)=a^{+}-a^{-}$and $L(b)=b^{+}-b^{-}$.

\section{A new distance measure between picture fuzzy sets}

Distance measure plays an important role in a lot of areas. Initially, many studies have focused on this issue on fuzzy sets, IFSs, NSs and so on, such as Euclidean distance and Hamming distance. Later, other extensions of the above distance measures were developed for picture fuzzy sets, such as the normalized picture Hamming distance, the normalized picture Euclidean distance and the generalized picture distance measure. Although the picture Hamming distance measure and the normalized picture Euclidean distance measure have their successful application in picture fuzzy clustering and multicriteria decision-making, some counter-intuitive phenomenon may occur in practical application. For example, let $A=(0.1,0.1,0), B=(0.2,0.2,0)$, and $C=(0.2,0.1,0)$ be three picture fuzzy sets. As we know, when the degree of neutral-membership is equal to zero, PFS reduces to IFS. Thus, $A, B$, and $C$ all belong to IFSs. According to the ranking function for the IFSs given in Definition 2, we have $A<B<C$, and the Hamming distance measure between $A$ and $C$ should be higher than that between $A$ and $B$. According to Definition 4, however, we have $d_{H}(A, B)=0.1, d_{H}(A, C)=0.05$, and $d_{H}(A, B)>d_{H}(A, C)$, which is against our intuition. To overcome this drawback, we propose a new biparametric distance measure for PFSs, which is a generalization of the biparametric intuitionistic distance measure proposed by Boran and Akay [5].

Definition 6. Given any two PFSs $A$ and B, the biparametric distance measure between PFSs $A$ and $B$ is defined as follows:

$$
\begin{aligned}
d(A, B) & =\left(\frac { 1 } { 3 n ( t + 1 ) ^ { P } } \sum _ { j = 1 } ^ { n } \left(\left|t\left(\mu_{A}\left(x_{j}\right)-\mu_{B}\left(x_{j}\right)\right)-\left(\nu_{A}\left(x_{j}\right)-\nu_{B}\left(x_{j}\right)\right)-\left(\gamma_{A}\left(x_{j}\right)-\gamma_{B}\left(x_{j}\right)\right)\right|^{P}\right.\right. \\
& +\sum_{j=1}^{n}\left|t\left(\nu_{A}\left(x_{j}\right)-\nu_{B}\left(x_{j}\right)\right)-\left(\mu_{A}\left(x_{j}\right)-\mu_{B}\left(x_{j}\right)\right)-\left(\gamma_{A}\left(x_{j}\right)-\gamma_{B}\left(x_{j}\right)\right)\right|^{P} \\
& \left.+\sum_{j=1}^{n}\left|t\left(\gamma_{A}\left(x_{j}\right)-\gamma_{B}\left(x_{j}\right)\right)-\left(\mu_{A}\left(x_{j}\right)-\mu_{B}\left(x_{j}\right)\right)-\left(\nu_{A}\left(x_{j}\right)-\nu_{B}\left(x_{j}\right)\right)\right|^{P}\right)^{1 / P}
\end{aligned}
$$

Where $t=2,3,4, \ldots$, parameter $t$ identifies the level of uncertainty.

The biparametric parametric picture distance measure has the capability of distinguishing positive difference from negative difference, and does not yield the counter-intuitive phenomena. For example, suppose $A=(0.1,0.1,0), B=(0.2,0.2,0)$, and $C=(0.2,0.1,0)$ be three PFSs. Let $t=2$ and $p=1$, according to Definition 5, we have $d(A, B)=0.017$ and $d(A, C)=0.033$. The distance measure between $A$ and $B$ is lower than that between $A$ and $C$. Meanwhile the measure is consistent with the fact that $A<B<C$. However, with Definition 4, we have $d_{H}(A, B)>d_{H}(A, C)$. This implies our proposed parametric distance measure is more reasonable than the normalized picture Hamming distance measure.

Theorem 7. $d(A, B)$ is a biparametric parametric distance between two PFSs $A$ and $B$ in $X$.

Proof. We prove Theorem 1 according to Definition 6 . We first show that $0 \leq d(A, B) \leq 1$. Note that

$$
\begin{array}{r}
\left|t\left(\mu_{A}\left(x_{j}\right)-\mu_{B}\left(x_{j}\right)\right)-\left(\nu_{A}\left(x_{j}\right)-\nu_{B}\left(x_{j}\right)\right)-\left(\gamma_{A}\left(x_{j}\right)-\gamma_{B}\left(x_{j}\right)\right)\right|= \\
\left|\left(t \mu_{A}\left(x_{j}\right)-\nu_{A}\left(x_{j}\right)-\gamma_{A}\left(x_{j}\right)\right)-\left(t \mu_{B}\left(x_{j}\right)-\nu_{B}\left(x_{j}\right)-\gamma_{B}\left(x_{j}\right)\right)\right|
\end{array}
$$




$$
\begin{array}{r}
\left|t\left(\nu_{A}\left(x_{j}\right)-\nu_{B}\left(x_{j}\right)\right)-\left(\mu_{A}\left(x_{j}\right)-\mu_{B}\left(x_{j}\right)\right)-\left(\gamma_{A}\left(x_{j}\right)-\gamma_{B}\left(x_{j}\right)\right)\right|= \\
\left|\left(t \nu_{A}\left(x_{j}\right)-\mu_{A}\left(x_{j}\right)-\gamma_{A}\left(x_{j}\right)\right)-\left(t \nu_{B}\left(x_{j}\right)-\mu_{B}\left(x_{j}\right)-\gamma_{B}\left(x_{j}\right)\right)\right| \\
\left|t\left(\gamma_{A}\left(x_{j}\right)-\gamma_{B}\left(x_{j}\right)\right)-\left(\mu_{A}\left(x_{j}\right)-\mu_{B}\left(x_{j}\right)\right)-\left(\nu_{A}\left(x_{j}\right)-\nu_{B}\left(x_{j}\right)\right)\right|= \\
\left|\left(t \gamma_{A}\left(x_{j}\right)-\mu_{A}\left(x_{j}\right)-\nu_{A}\left(x_{j}\right)\right)-\left(t \gamma_{B}\left(x_{j}\right)-\mu_{B}\left(x_{j}\right)-\nu_{B}\left(x_{j}\right)\right)\right|
\end{array}
$$

since $\mu_{A}(x), \nu_{A}(x), \gamma_{A}(x) \in[0,1]$, and $\mu_{A}(x)+\nu_{A}(x)+\gamma_{A}(x) \in[0,1]$, we have the following inequations:

$$
\begin{gathered}
-1 \leq\left(t \mu_{A}\left(x_{i}\right)-\nu_{A}\left(x_{i}\right)-\gamma_{A}\left(x_{i}\right)\right) \leq t \\
-t \leq-\left(t \mu_{B}\left(x_{i}\right)-\nu_{B}\left(x_{i}\right)-\gamma_{B}\left(x_{i}\right)\right) \leq 1
\end{gathered}
$$

Then we have

$$
-(t+1) \leq\left(t \mu_{A}\left(x_{j}\right)-\nu_{A}\left(x_{j}\right)-\gamma_{A}\left(x_{j}\right)\right)-\left(t \mu_{B}\left(x_{j}\right)-\nu_{B}\left(x_{j}\right)-\gamma_{B}\left(x_{j}\right)\right) \leq t+1
$$

This implies

$$
0 \leq\left|\left(t \mu_{A}\left(x_{j}\right)-\nu_{A}\left(x_{j}\right)-\gamma_{A}\left(x_{j}\right)\right)-\left(t \mu_{B}\left(x_{j}\right)-\nu_{B}\left(x_{j}\right)-\gamma_{B}\left(x_{j}\right)\right)\right|^{p} \leq(t+1)^{p}
$$

Similarly, we have the following inequations:

$$
\begin{aligned}
& 0 \leq\left|\left(t \nu_{A}\left(x_{j}\right)-\mu_{A}\left(x_{j}\right)-\gamma_{A}\left(x_{j}\right)\right)-\left(t \nu_{B}\left(x_{j}\right)-\mu_{B}\left(x_{j}\right)-\gamma_{B}\left(x_{j}\right)\right)\right|^{p} \leq(t+1)^{p} \\
& 0 \leq\left|\left(t \gamma_{A}\left(x_{j}\right)-\mu_{A}\left(x_{j}\right)-\nu_{A}\left(x_{j}\right)\right)-\left(t \gamma_{B}\left(x_{j}\right)-\mu_{B}\left(x_{j}\right)-\nu_{B}\left(x_{j}\right)\right)\right|^{p} \leq(t+1)^{p}
\end{aligned}
$$

Finally, we have the following inequations:

$$
\begin{aligned}
0 \leq & \left(\frac { 1 } { 3 n ( t + 1 ) ^ { P } } \sum _ { j = 1 } ^ { n } \left(\left|\left(t \mu_{A}\left(x_{j}\right)-\mu_{B}\left(x_{j}\right)\right)-\left(\nu_{A}\left(x_{j}\right)-\nu_{B}\left(x_{j}\right)\right)-\left(\gamma_{A}\left(x_{j}\right)-\gamma_{B}\left(x_{j}\right)\right)\right|^{P}\right.\right. \\
& +\left|t\left(\nu_{A}\left(x_{j}\right)-\nu_{B}\left(x_{j}\right)\right)-\left(\mu_{A}\left(x_{j}\right)-\mu_{B}\left(x_{j}\right)\right)-\left(\gamma_{A}\left(x_{j}\right)-\gamma_{B}\left(x_{j}\right)\right)\right|^{P} \\
& \left.\left.+\left|t\left(\gamma_{A}\left(x_{j}\right)-\gamma_{B}\left(x_{j}\right)\right)-\left(\mu_{A}\left(x_{j}\right)-\mu_{B}\left(x_{j}\right)\right)-\left(\nu_{A}\left(x_{j}\right)-\nu_{B}\left(x_{j}\right)\right)\right|^{P}\right)\right)^{1 / P}
\end{aligned}
$$

Thus, $0 \leq d(A, B) \leq 1$

Now we prove that $d(A, B)=0$ if $A=B$. If $\mu_{A}\left(x_{j}\right)=\mu_{B}\left(x_{j}\right), \nu_{A}\left(x_{j}\right)=\nu_{B}\left(x_{j}\right)$ and $\gamma_{A}\left(x_{j}\right)=$ $\gamma_{B}\left(x_{j}\right)$, we have $\mu_{A}\left(x_{j}\right)-\mu_{B}\left(x_{j}\right)=0, \nu_{A}\left(x_{j}\right)-\nu_{B}\left(x_{j}\right)=0$ and $\gamma_{A}\left(x_{j}\right)-\gamma_{B}\left(x_{j}\right)=0$. Thus, the distance measure $d(A, B)$ is equal to zero.

We now prove that $d(A, B)=d(B, A)$. Note that

$$
\begin{aligned}
& \left|t\left(\mu_{A}\left(x_{j}\right)-\mu_{B}\left(x_{j}\right)\right)-\left(\nu_{A}\left(x_{j}\right)-\nu_{B}\left(x_{j}\right)\right)-\left(\gamma_{A}\left(x_{j}\right)-\gamma_{B}\left(x_{j}\right)\right)\right|^{P}= \\
& \left|(-1)\left\{t\left(\mu_{B}\left(x_{j}\right)-\mu_{A}\left(x_{j}\right)\right)-\left(\nu_{B}\left(x_{j}\right)-\nu_{A}\left(x_{j}\right)\right)-\left(\gamma_{B}\left(x_{j}\right)-\gamma_{A}\left(x_{j}\right)\right)\right\}\right|^{P} ; \\
& \left|t\left(\nu_{A}\left(x_{j}\right)-\nu_{B}\left(x_{j}\right)\right)-\left(\mu_{A}\left(x_{j}\right)-\mu_{B}\left(x_{j}\right)\right)-\left(\gamma_{A}\left(x_{j}\right)-\gamma_{B}\left(x_{j}\right)\right)\right|^{P} \\
& =\left|(-1)\left\{t\left(\nu_{B}\left(x_{j}\right)-\nu_{A}\left(x_{j}\right)\right)-\left(\mu_{B}\left(x_{j}\right)-\mu_{A}\left(x_{j}\right)\right)-\left(\gamma_{B}\left(x_{j}\right)-\gamma_{A}\left(x_{j}\right)\right)\right\}\right|^{P} \\
& \left|t\left(\gamma_{A}\left(x_{j}\right)-\gamma_{B}\left(x_{j}\right)\right)-\left(\mu_{A}\left(x_{j}\right)-\mu_{B}\left(x_{j}\right)\right)-\left(\nu_{A}\left(x_{j}\right)-\nu_{B}\left(x_{j}\right)\right)\right|^{P} \\
& =\left|(-1)\left\{t\left(\gamma_{B}\left(x_{j}\right)-\gamma_{A}\left(x_{j}\right)\right)-\left(\mu_{B}\left(x_{j}\right)-\mu_{A}\left(x_{j}\right)\right)-\left(\nu_{B}\left(x_{j}\right)-\nu_{A}\left(x_{j}\right)\right)\right\}\right|^{P}
\end{aligned}
$$

Based on definition of absolute value, we have

$$
\begin{aligned}
& \left|t\left(\mu_{A}\left(x_{j}\right)-\mu_{B}\left(x_{j}\right)\right)-\left(\nu_{A}\left(x_{j}\right)-\nu_{B}\left(x_{j}\right)\right)-\left(\gamma_{A}\left(x_{j}\right)-\gamma_{B}\left(x_{j}\right)\right)\right|^{P} \\
& =\left|t\left(\mu_{B}\left(x_{j}\right)-\mu_{A}\left(x_{j}\right)\right)-\left(\nu_{B}\left(x_{j}\right)-\nu_{A}\left(x_{j}\right)\right)-\left(\gamma_{B}\left(x_{j}\right)-\gamma_{A}\left(x_{j}\right)\right)\right|^{P} \\
& \left|t\left(\gamma_{A}\left(x_{j}\right)-\gamma_{B}\left(x_{j}\right)\right)-\left(\mu_{A}\left(x_{j}\right)-\mu_{B}\left(x_{j}\right)\right)-\left(\nu_{A}\left(x_{j}\right)-\nu_{B}\left(x_{j}\right)\right)\right|^{P} \\
& =\left|t\left(\gamma_{B}\left(x_{j}\right)-\gamma_{A}\left(x_{j}\right)\right)-\left(\mu_{B}\left(x_{j}\right)-\mu_{A}\left(x_{j}\right)\right)-\left(\nu_{B}\left(x_{j}\right)-\nu_{A}\left(x_{j}\right)\right)\right|^{P} \\
& \left|t\left(\nu_{A}\left(x_{j}\right)-\nu_{B}\left(x_{j}\right)\right)-\left(\mu_{A}\left(x_{j}\right)-\mu_{B}\left(x_{j}\right)\right)-\left(\gamma_{A}\left(x_{j}\right)-\gamma_{B}\left(x_{j}\right)\right)\right|^{P} \\
& =\left|t\left(\nu_{B}\left(x_{j}\right)-\nu_{A}\left(x_{j}\right)\right)-\left(\mu_{B}\left(x_{j}\right)-\mu_{A}\left(x_{j}\right)\right)-\left(\gamma_{B}\left(x_{j}\right)-\gamma_{A}\left(x_{j}\right)\right)\right|^{P}
\end{aligned}
$$


Thus, $d(A, B)=d(B, A)$. We now prove the fourth condition of Definition 6. By Definition 6, the distance measures $d(A, B)$ and $d(A, C)$ are respectively given as follows:

$$
\begin{aligned}
d(A, B) & =\left(\frac { 1 } { 3 n ( t + 1 ) ^ { P } } \sum _ { j = 1 } ^ { n } \left(\left|t\left(\mu_{A}\left(x_{j}\right)-\mu_{B}\left(x_{j}\right)\right)-\left(\nu_{A}\left(x_{j}\right)-\nu_{B}\left(x_{j}\right)\right)-\left(\gamma_{A}\left(x_{j}\right)-\gamma_{B}\left(x_{j}\right)\right)\right|^{P}\right.\right. \\
& +\left|t\left(\nu_{A}\left(x_{j}\right)-\nu_{B}\left(x_{j}\right)\right)-\left(\mu_{A}\left(x_{j}\right)-\mu_{B}\left(x_{j}\right)\right)-\left(\gamma_{A}\left(x_{j}\right)-\gamma_{B}\left(x_{j}\right)\right)\right|^{P} \\
& \left.\left.+\left|t\left(\gamma_{A}\left(x_{j}\right)-\gamma_{B}\left(x_{j}\right)\right)-\left(\mu_{A}\left(x_{j}\right)-\mu_{B}\left(x_{j}\right)\right)-\left(\nu_{A}\left(x_{j}\right)-\nu_{B}\left(x_{j}\right)\right)\right|^{P}\right)\right)^{1 / P} \\
d(A, C) & =\left(\frac { 1 } { 3 n ( t + 1 ) ^ { P } } \sum _ { j = 1 } ^ { n } \left(\left|t\left(\mu_{A}\left(x_{j}\right)-\mu_{C}\left(x_{j}\right)\right)-\left(\nu_{A}\left(x_{j}\right)-\nu_{C}\left(x_{j}\right)\right)-\left(\gamma_{A}\left(x_{j}\right)-\gamma_{C}\left(x_{j}\right)\right)\right|^{P}\right.\right. \\
& +\left|t\left(\nu_{A}\left(x_{j}\right)-\nu_{C}\left(x_{j}\right)\right)-\left(\mu_{A}\left(x_{j}\right)-\mu_{C}\left(x_{j}\right)\right)-\left(\gamma_{A}\left(x_{j}\right)-\gamma_{C}\left(x_{j}\right)\right)\right|^{P} \\
& \left.\left.+\left|t\left(\gamma_{A}\left(x_{j}\right)-\gamma_{C}\left(x_{j}\right)\right)-\left(\mu_{A}\left(x_{j}\right)-\mu_{C}\left(x_{j}\right)\right)-\left(\nu_{A}\left(x_{j}\right)-\nu_{C}\left(x_{j}\right)\right)\right|^{P}\right)\right)^{1 / P}
\end{aligned}
$$

If $A \subseteq B \subseteq C$, according to Definition 6 we have $0 \leq \mu_{A}\left(x_{j}\right) \leq \mu_{B}\left(x_{j}\right) \leq \mu_{C}\left(x_{j}\right) \leq 1,0 \leq$ $\nu_{C}\left(x_{j}\right) \leq \nu_{B}\left(x_{j}\right) \leq \nu_{A}\left(x_{j}\right) \leq 1$ and $0 \leq \gamma_{C}\left(x_{j}\right) \leq \gamma_{B}\left(x_{j}\right) \leq \gamma_{A}\left(x_{j}\right) \leq 1$. Then we obtain the following inequations:

$$
\begin{aligned}
& \left|t\left(\mu_{A}\left(x_{j}\right)-\mu_{C}\left(x_{j}\right)\right)-\left(\nu_{A}\left(x_{j}\right)-\nu_{C}\left(x_{j}\right)\right)-\left(\gamma_{A}\left(x_{j}\right)-\gamma_{C}\left(x_{j}\right)\right)\right|^{P} \\
& =\left(\mid t\left(\mu_{A}\left(x_{j}\right)-\mu_{C}\left(x_{j}\right)\right)-\left(\nu_{A}\left(x_{j}\right)-\nu_{C}\left(x_{j}\right)\right)-\left(\gamma_{A}\left(x_{j}\right)-\gamma_{C}\left(x_{j}\right)\right)\right. \\
& \left.\quad+t \mu_{B}\left(x_{j}\right)-t \mu_{B}\left(x_{j}\right)+\nu_{B}\left(x_{j}\right)-\nu_{B}\left(x_{j}\right)+\gamma_{B}\left(x_{j}\right)-\gamma_{B}\left(x_{j}\right) \mid\right)^{P} \\
& =\left(\mid\left[t\left(\mu_{A}\left(x_{j}\right)-\mu_{B}\left(x_{j}\right)\right)-\left(\nu_{A}\left(x_{j}\right)-\nu_{B}\left(x_{j}\right)\right)-\left(\gamma_{A}\left(x_{j}\right)-\gamma_{B}\left(x_{j}\right)\right)\right]\right. \\
& {\left[t\left(\mu_{B}\left(x_{j}\right)-\mu_{C}\left(x_{j}\right)\right)-\left(\nu_{B}\left(x_{j}\right)-\nu_{C}\left(x_{j}\right)\right)-\left(\gamma_{B}\left(x_{j}\right)-\gamma_{C}\left(x_{j}\right)\right] \mid\right)^{p}} \\
& \leq\left(\mid t\left(\mu_{A}\left(x_{j}\right)-\mu_{B}\left(x_{j}\right)\right)-\left(\nu_{A}\left(x_{j}\right)-\nu_{B}\left(x_{j}\right)\right)-\left(\gamma_{A}\left(x_{j}\right)-\gamma_{B}\left(x_{j}\right) \mid\right)\right. \\
& \left.+\left|t\left(\mu_{B}\left(x_{j}\right)-\mu_{C}\left(x_{j}\right)\right)-\left(\nu_{B}\left(x_{j}\right)-\nu_{C}\left(x_{j}\right)\right)-\left(\gamma_{B}\left(x_{j}\right)-\gamma_{C}\left(x_{j}\right)\right)\right|\right)^{p}
\end{aligned}
$$

Similarly, we have the following inequations:

$$
\begin{aligned}
& \left|t\left(\nu_{A}\left(x_{j}\right)-\nu_{C}\left(x_{j}\right)\right)-\left(\mu_{A}\left(x_{j}\right)-\mu_{C}\left(x_{j}\right)\right)-\left(\gamma_{A}\left(x_{j}\right)-\gamma_{C}\left(x_{j}\right)\right)\right|^{P} \\
\leq & \left(\mid t\left(\mu_{A}\left(x_{j}\right)-\mu_{B}\left(x_{j}\right)\right)-\left(\nu_{A}\left(x_{j}\right)-\nu_{B}\left(x_{j}\right)\right)-\left(\gamma_{A}\left(x_{j}\right)-\gamma_{B}\left(x_{j}\right) \mid\right)\right. \\
+ & \left.\left|t\left(\nu_{B}\left(x_{j}\right)-\nu_{C}\left(x_{j}\right)\right)-\left(\mu_{B}\left(x_{j}\right)-\mu_{C}\left(x_{j}\right)\right)-\left(\gamma_{B}\left(x_{j}\right)-\gamma_{C}\left(x_{j}\right)\right)\right|^{P}\right) \\
& \left|t\left(\gamma_{A}\left(x_{j}\right)-\gamma_{C}\left(x_{j}\right)\right)-\left(\mu_{A}\left(x_{j}\right)-\mu_{C}\left(x_{j}\right)\right)-\left(\nu_{A}\left(x_{j}\right)-\nu_{C}\left(x_{j}\right)\right)\right| \\
\leq & \left|t\left(\gamma_{A}\left(x_{j}\right)-\gamma_{B}\left(x_{j}\right)\right)-\left(\mu_{A}\left(x_{j}\right)-\mu_{B}\left(x_{j}\right)\right)-\left(\nu_{A}\left(x_{j}\right)-\nu_{B}\left(x_{j}\right)\right)\right| \\
+ & \left.\left|t\left(\gamma_{B}\left(x_{j}\right)-\gamma_{C}\left(x_{j}\right)\right)-\left(\mu_{B}\left(x_{j}\right)-\mu_{C}\left(x_{j}\right)\right)-\left(\nu_{B}\left(x_{j}\right)-\nu_{C}\left(x_{j}\right)\right)\right|^{P}\right)
\end{aligned}
$$

And then, we have the following inequations:

$$
\begin{aligned}
& d^{P}(A, C)=\left(\frac { 1 } { 3 n ( t + 1 ) ^ { P } } \sum _ { j = 1 } ^ { n } \left(\left|t\left(\mu_{A}\left(x_{j}\right)-\mu_{C}\left(x_{j}\right)\right)-\left(\nu_{A}\left(x_{j}\right)-\nu_{C}\left(x_{j}\right)\right)-\left(\gamma_{A}\left(x_{j}\right)-\gamma_{C}\left(x_{j}\right)\right)\right|^{P}\right.\right. \\
&+\left|t\left(\nu_{A}\left(x_{j}\right)-\nu_{C}\left(x_{j}\right)\right)-\left(\mu_{A}\left(x_{j}\right)-\mu_{C}\left(x_{j}\right)\right)-\left(\gamma_{A}\left(x_{j}\right)-\gamma_{C}\left(x_{j}\right)\right)\right|^{P} \\
&+\left.t\left(\gamma_{A}\left(x_{j}\right)-\gamma_{C}\left(x_{j}\right)\right)-\left(\mu_{A}\left(x_{j}\right)-\mu_{C}\left(x_{j}\right)\right)-\left.\left(\nu_{A}\left(x_{j}\right)-\nu_{C}\left(x_{j}\right)\right)\right|^{P}\right) \\
& \leq \frac{1}{3 n(t+1)^{P}} \sum_{j=1}^{n}\left(\left|t\left(\mu_{A}\left(x_{j}\right)-\mu_{B}\left(x_{j}\right)\right)-\left(\nu_{A}\left(x_{j}\right)-\nu_{B}\left(x_{j}\right)\right)-\left(\gamma_{A}\left(x_{j}\right)-\gamma_{B}\left(x_{j}\right)\right)\right|\right) \\
&\left.\quad+\left|t\left(\mu_{B}\left(x_{j}\right)-\mu_{C}\left(x_{j}\right)\right)-\left(\nu_{B}\left(x_{j}\right)-\nu_{C}\left(x_{j}\right)\right)-\left(\gamma_{B}\left(x_{j}\right)-\gamma_{C}\left(x_{j}\right)\right)\right|\right)^{p} \\
&+\frac{1}{3 n(t+1)^{P}} \sum_{j=1}^{n}\left(\left|t\left(\nu_{A}\left(x_{j}\right)-\nu_{B}\left(x_{j}\right)\right)-\left(\mu_{A}\left(x_{j}\right)-\mu_{B}\left(x_{j}\right)\right)-\left(\gamma_{A}\left(x_{j}\right)-\gamma_{B}\left(x_{j}\right)\right)\right|\right) \\
&\left.\quad+\left|t\left(\nu_{B}\left(x_{j}\right)-\nu_{C}\left(x_{j}\right)\right)-\left(\mu_{B}\left(x_{j}\right)-\mu_{C}\left(x_{j}\right)\right)-\left(\gamma_{B}\left(x_{j}\right)-\gamma_{C}\left(x_{j}\right)\right)\right|^{P}\right)
\end{aligned}
$$




$$
\begin{aligned}
& +\frac{1}{3 n(t+1)^{P}} \sum_{j=1}^{n}\left(\left|t\left(\gamma_{A}\left(x_{j}\right)-\gamma_{B}\left(x_{j}\right)\right)-\left(\mu_{A}\left(x_{j}\right)-\mu_{B}\left(x_{j}\right)\right)-\left(\nu_{A}\left(x_{j}\right)-\nu_{B}\left(x_{j}\right)\right)\right|\right) \\
& \left.\quad+\left|t\left(\gamma_{B}\left(x_{j}\right)-\gamma_{C}\left(x_{j}\right)\right)-\left(\mu_{B}\left(x_{j}\right)-\mu_{C}\left(x_{j}\right)\right)-\left(\nu_{B}\left(x_{j}\right)-\nu_{C}\left(x_{j}\right)\right)\right|^{P}\right) \\
& \leq \frac{1}{3 n(t+1)^{P}} \sum_{j=1}^{n}\left[\left(\left|t\left(\mu_{A}\left(x_{j}\right)-\mu_{B}\left(x_{j}\right)\right)-\left(\nu_{A}\left(x_{j}\right)-\nu_{B}\left(x_{j}\right)\right)-\left(\gamma_{A}\left(x_{j}\right)-\gamma_{B}\left(x_{j}\right)\right)\right|\right.\right. \\
& \quad+\left|t\left(\mu_{B}\left(x_{j}\right)-\mu_{C}\left(x_{j}\right)\right)-\left(\nu_{B}\left(x_{j}\right)-\nu_{C}\left(x_{j}\right)\right)-\left(\gamma_{B}\left(x_{j}\right)-\gamma_{C}\left(x_{j}\right)\right)\right| \\
& \quad+\left(\left|t\left(\nu_{A}\left(x_{j}\right)-\nu_{B}\left(x_{j}\right)\right)-\left(\mu_{A}\left(x_{j}\right)-\mu_{B}\left(x_{j}\right)\right)-\left(\gamma_{A}\left(x_{j}\right)-\gamma_{B}\left(x_{j}\right)\right)\right|\right) \\
& \left.\quad+\left|t\left(\nu_{B}\left(x_{j}\right)-\nu_{C}\left(x_{j}\right)\right)-\left(\mu_{B}\left(x_{j}\right)-\mu_{C}\left(x_{j}\right)\right)-\left(\gamma_{B}\left(x_{j}\right)-\gamma_{C}\left(x_{j}\right)\right)\right|\right) \\
& \quad+\left(\left|t\left(\gamma_{A}\left(x_{j}\right)-\gamma_{B}\left(x_{j}\right)\right)-\left(\mu_{A}\left(x_{j}\right)-\mu_{B}\left(x_{j}\right)\right)-\left(\nu_{A}\left(x_{j}\right)-\nu_{B}\left(x_{j}\right)\right)\right|\right) \\
& \left.+\left|t\left(\gamma_{B}\left(x_{j}\right)-\gamma_{C}\left(x_{j}\right)\right)-\left(\mu_{B}\left(x_{j}\right)-\mu_{C}\left(x_{j}\right)\right)-\left(\nu_{B}\left(x_{j}\right)-\nu_{C}\left(x_{j}\right)\right)\right|\right]^{P} \\
& \leq(d(A, B)+d(B, C))^{P}
\end{aligned}
$$

We know that $d(A, C) \geq 0, d(A, B) \geq 0$, and $d(B, C) \geq 0$, and therefore we have the following inequalities:

$$
d(A, C) \leq d(A, B)+d(B, C)
$$

We thus prove that $d(A, B)$ is a biparametric parametric distance measure between picture fuzzy sets $A$ and $B$, since $d(A, B)$ satisfies Definition 3 .

Definition 8. Given any two PFVs $\alpha_{1}$ and $\alpha_{2}$, a biparametric distance measure between $\alpha_{1}$ and $\alpha_{2}$ is defined as follows:

$$
\begin{aligned}
d\left(\alpha_{1}, \alpha_{2}\right)= & \left(\frac { 1 } { 3 ( t + 1 ) ^ { P } } \left(\left|t\left(\mu_{\alpha_{1}}-\mu_{\alpha_{2}}\right)-\left(\nu_{\alpha_{1}}-\nu_{\alpha_{2}}\right)-\left(\gamma_{\alpha_{1}}-\gamma_{\alpha_{2}}\right)\right|^{P}\right.\right. \\
& +\left|t\left(\nu_{\alpha_{1}}-\nu_{\alpha_{2}}\right)-\left(\mu_{\alpha_{1}}-\mu_{\alpha_{2}}\right)-\left(\gamma_{\alpha_{1}}-\gamma_{\alpha_{2}}\right)\right|^{P} \\
& \left.+\left|t\left(\gamma_{\alpha_{1}}-\gamma_{\alpha_{2}}\right)-\left(\nu_{\alpha_{1}}-\nu_{\alpha_{2}}\right)-\left(\mu_{\alpha_{1}}-\mu_{\alpha_{2}}\right)\right|^{P}\right)^{1 / P}
\end{aligned}
$$

where $t=2,3,4, \cdots$, parameter, and $t$ identifies the level of uncertainty.

\section{Fractional programming models for solving MCDM problems with PFSs environments}

For a MCMD problem under picture fuzzy environment, let $C=\left(C_{1}, C_{2}, \cdots, C_{n}\right)$ be a set of criteria, and $X=\left(X_{1}, X_{2}, \cdots, X_{m}\right)$ be a set of alternatives to be selected. Let $X=\left(\alpha_{i j}\right)_{m \times n}$ be a picture fuzzy decision matrix, where $\alpha_{i j}=\left(\mu_{\alpha_{i j}}, \nu_{\alpha_{i j}}, \gamma_{\alpha_{i j}}\right)$ is a PFV for alternative $X_{i}$ with respect to criteria $C_{j}$ provided by decision-maker, such that $0 \leq \mu_{\alpha_{i j}} \leq 1,0 \leq \nu_{\alpha_{i j}} \leq 1,0 \leq \gamma_{\alpha_{i j}} \leq 1$, $0 \leq \mu_{\alpha_{i j}}+\nu_{\alpha_{i j}}+\gamma_{\alpha_{i j}} \leq 1, i=1,2, \cdots, m$ and $j=1,2, \cdots, n$. Therefore, a positive ideal solution $X^{+}$ and a negative ideal solution $X^{-}$may be defined in the following way. The degree $\bar{\mu}_{\alpha_{i j}}$ of positive membership, the degree $\bar{\nu}_{\alpha_{i j}}$ of negative membership, and the degree $\bar{\gamma}_{\alpha_{i j}}$ of neutral membership with respect to attribute $C_{j} \in C$ are 1 and 0 , respectively, which can be written as $\alpha_{i j}^{+}=\left(\mu_{\alpha_{i j}}^{+}, \nu_{\alpha_{i j}}^{+}, \gamma_{\alpha_{i j}}^{+}\right)=$ $(1,0,0)$ and $\alpha_{i j}^{-}=\left(\mu_{\alpha_{i j}}^{-}, \nu_{\alpha_{i j}}^{-}, \gamma_{\alpha_{i j}}^{-}\right)=(0,1,0)$. Then, the distance matrix can be given as $D^{+}=\left(d_{i j}^{+}\right)$, and $D^{-}=\left(d_{i j}^{-}\right)$, where $d_{i j}^{+}$is the distance between $\alpha_{i j}^{+}$and $\alpha_{i j}$, and $d_{i j}^{-}$is the distance between $\alpha_{i j}^{-}$and $\alpha_{i j}$, respectively. Assumed that the decision maker constructs a PFS $W_{j}=\left(A_{j}, \omega_{j}\right)$, where $\omega_{j}$ is the weight of the criteria $C_{j} \in C$ on the fuzzy concept "importance", where $\omega_{j} \in[0,1]$, and $\sum_{j=1}^{n} \varpi_{j}=1$. The importance indices allow us to calculate the best final result as well as the smallest one, which we can expect in a process leading to a final decision. During the making decision process, the decision maker may change his/her evaluating weights by adjusting the value of the weight index. So in fact his/her weight lies in the closed interval $\omega_{j}=\left[\omega_{j}^{l}, \omega_{j}^{u}\right]$, where $0 \leq \omega_{j}^{l} \leq \omega_{j}^{u} \leq 1$. Based on TOPSIS, the relative-closeness coefficient of each $X_{j} \in X,(i=1,2, \cdots, m)$ with respect to $X_{i}^{+}$is defined as follows: 


$$
C_{i}=\frac{\sqrt{\sum_{j=1}^{n}\left(\omega_{j} d_{i j}^{+}\right)^{2}}}{\sqrt{\sum_{j=1}^{n}\left(\omega_{j} d_{i j}^{+}\right)^{2}}+\sqrt{\sum_{j=1}^{n}\left(\omega_{j} d_{i j}^{-}\right)^{2}}}
$$

Where $\omega_{j}^{l} \leq \omega_{j} \leq \omega_{j}^{u}$ for all $i=1,2, \cdots, m$ and $j=1,2, \cdots, n$.

Obviously, $C_{i}$ is different for different $\omega_{j} \in\left[\omega_{j}^{l}, \omega_{j}^{u}\right]$. Values of $C_{i}$ should be in some range when $C_{i}$ takes all values in the interval $\left[\omega_{j}^{l}, \omega_{j}^{u}\right]$. In other words, $C_{i}$ is an interval number, denoted by $R_{j}=\left[C_{j}^{l}, C_{j}^{u}\right]$. The lower and upper bounds $C_{j}^{l}$ and $C_{j}^{u}$ of $C_{i}$ can be captured by solving the following pair of fractional programming models:

$$
\begin{gathered}
C_{i}^{l}=\min _{i}=\frac{\sqrt{\sum_{j=1}^{n}\left(\omega_{j} d_{i j}^{+}\right)^{2}}}{\sqrt{\sum_{j=1}^{n}\left(\omega_{j} d_{i j}^{+}\right)^{2}}+\sqrt{\sum_{j=1}^{n}\left(\omega_{j} d_{i j}^{-}\right)^{2}}} \\
\text { s.t. } C_{j}^{l}, \leq C_{j} \leq C_{j}^{u} ; j=1,2, \cdots, n
\end{gathered}
$$

, and

$$
\begin{gathered}
C_{i}^{l}=\max C_{i}=\frac{\sqrt{\sum_{j=1}^{n}\left(\omega_{j} d_{i j}^{+}\right)^{2}}}{\sqrt{\sum_{j=1}^{n}\left(\omega_{j} d_{i j}^{+}\right)^{2}}+\sqrt{\sum_{j=1}^{n}\left(\omega_{j} d_{i j}^{-}\right)^{2}}} \\
\text { s.t. } C_{j}^{l}, \leq C_{j} \leq C_{j}^{u} ; j=1,2, \cdots, n
\end{gathered}
$$

Due to the fact that

$$
\frac{\partial C_{i}}{\partial \omega_{j}}=\frac{\left(\omega_{j}\right)^{2} d_{i j}^{+} \sqrt{\sum_{j=1}^{n}\left(\omega_{j} d_{i j}^{-}\right)^{2} / \sum_{j=1}^{n}\left(\omega_{j} d_{i j}^{+}\right)^{2}}+\left(\omega_{j}\right)^{2} d_{i j}^{-} \sqrt{\sum_{j=1}^{n}\left(\omega_{j} d_{i j}^{+}\right)^{2} / \sum_{j=1}^{n}\left(\omega_{j} d_{i j}^{-}\right)^{2}}}{\left(\sqrt{\sum_{j=1}^{n}\left(\omega_{j} d_{i j}^{+}\right)^{2}}+\sqrt{\sum_{j=1}^{n}\left(\omega_{j} d_{i j}^{-}\right)^{2}}\right)^{2}} \geq 0
$$

Therefore, for $j=1,2, \cdots, n, C_{i}$ is a monotonically increasing function of $\omega_{i j}$, which means that $C_{j}$ reaches its maximum at $\omega_{j}^{u}$ and arrives at its minimum at $\omega_{j}^{l}$.

Then, the relative closeness coefficient interval $R_{j}=\left[C_{j}^{l}, C_{j}^{u}\right]$ of the alternative $X_{i} \in X$ for the decision-maker can be obtained by solving Eqs. (8) and (9) using the existing optimization software such as Lingo and Excel. Later, using Eq. (4), the likelihood of $X_{s} \succ X_{t}$ for alternatives $X_{s}$ and $X_{t}$ in $X$ can be determined as follows:

$$
P\left(X_{s} \succ X_{t}\right)=P\left(C_{s} \succ C_{t}\right)=\max \left\{\left(1-\max \left(\frac{C_{t}^{u}-C_{s}^{l}}{L\left(C_{s}\right)+L\left(C_{t}\right)}\right), 0\right), 0\right\}
$$

where $L\left(C_{s}\right)=C_{j}^{u}-C_{j}^{l}$ and $L\left(C_{t}\right)=C_{j}^{u}-C_{j}^{l}$. Then the likelihood matrix can be obtained as follows:

$$
P=P\left(p_{s t}\right)_{m \times n}=\left[\begin{array}{cccc}
p_{11} & p_{12} & \cdots & p_{1 m} \\
p_{21} & p_{22} & \cdots & p_{2 m} \\
\vdots & \vdots & \cdots & \vdots \\
p_{m 1} & p_{m 2} & \cdots & p_{m m}
\end{array}\right]
$$

Where $p_{s t}=\left(P_{s} \geq P_{t}\right)$ for alternatives $X_{s}$ and $X_{t}$ in $X$. Thus, an optimal degree of membership for alternative $X_{i} \in X, i=1,2, \cdots, m$ is defined as follows [30]:

$$
\operatorname{rank}\left(X_{i}\right)=\frac{1}{m(m-1)}\left(\sum_{s=1}^{m} p_{i s}+\frac{m}{2}-1\right)
$$

Obviously, $\operatorname{rank}\left(X_{i}\right) \in[0,1]$ for alternative $X_{i} \in X, i=1,2, \cdots, m$, and $X_{s} \geq X_{t}$ if and only if $\operatorname{rank}\left(X_{s}\right) \geq \operatorname{rank}\left(X_{t}\right)$. 
The complete procedure for multi-criteria decision making based on the picture fuzzy sets and fractional programming models can be summarized as follows:

Step 1: Obtain the performance data in the form of picture fuzzy sets $\alpha_{i j}=\left(\mu_{\alpha_{i j}}, \nu_{\alpha_{i j}}, \gamma_{\alpha_{i j}}\right)$ $(i=1,2, \cdots, m ; j=1,2, \cdots, n)$ for $m$ alternatives over $n$ criteria and establish a picture fuzzy decision matrix $X=\left(\alpha_{i j}\right)_{m \times n}$.

Step 2: Transform the criteria values of cost type into those of benefit type. i.e., transform $\widetilde{X}=\left(\tilde{\alpha}_{i j}\right)_{m \times n}$ into the normalized picture fuzzy decision matrix $\widetilde{R}=\left(\tilde{r}_{i j}\right)_{m \times n}$, where

$$
\tilde{r}_{i j}=\left\{\begin{aligned}
\alpha_{i j}, & \text { for benefit criteria } \\
\left(\alpha_{i j}\right)^{c}, & \text { for cost criteria }
\end{aligned}\right.
$$

where $\left(\alpha_{i j}\right)^{c}$ is the complement of $\alpha_{i j}$ such that $\left(\alpha_{i j}\right)^{c}=\left(\nu_{i j}, \mu_{i j}, \gamma_{i j}\right)$.

Step 3: Identify the picture fuzzy positive ideal solution (PFPIS) and picture fuzzy negative ideal solution (PFNIS) based on the PFSs, and calculate the positive / negative picture fuzzy distance matrix $D^{+}=\left(d_{j}^{+}\right)$and $D^{-}=\left(d_{j}^{-}\right)$.

Step 4: Determine the relative closeness coefficient of each alternative by using Eqs. (8) and (9).

Step 5: Determine the likelihood of $X_{s} \succ X_{t}$ for alternatives $X_{s}$ and $X_{t}$ in $X$.

Step 6: Calculate the optimal degree of membership for alternative, and rank the alternatives according to optimal degree $\operatorname{rank}\left(X_{i}\right)$.

\section{A numerical example and comparison with the results of other method}

\subsection{A numerical example}

In this section, a numerical example adapted from [24] is worked out to illustrate the proposed method.

A company located in Ankara wants to select a best green supplier, where there are four alternatives to be selected: X1, X2, X3, and X4. X1 is located in the Marmara Region and exports to 4 countries. $\mathrm{X} 2$ is located in the Marmara Region and exports to 2 countries. X3 is located in the Central Anatolia Region. Moreover, X4 is located in the Marmara Region and exports to 6 countries. The four alternatives currently have approximately 200-500 employees. The team of decision makers is composed of experts from the finance department, engineering department and purchasing department. They believe that the information and data about performance of the green supplier can be reported using fuzzy approach. After experts' preliminary screening, 5 criteria are determined, such as Quality (C1), Cost (C2), Service and Delivery(C3), Sustainability (C4), and Environmental Management and Control (C5). To obtain an optimal green supplier, the following steps are given as follows.

Step 1. To select an ideal green supplier, the team of decision makers has agreed to assess each criteria $\mathrm{C}_{j}(j=1,2, \cdots, 5)$ of alternative $X_{i}(i=1,2,3,4)$ under picture fuzzy environment and constructs a decision matrix $X=\left(a_{i j}\right)_{4 \times 5}$, which are shown in Tab.1. The importance of each attribute $\mathrm{C}_{j},(j=1,2, \cdots, 5)$ are given in the form of intervals weight vectors as $\omega_{1}=[0.15,0.83], \omega_{2}=$ $[0.26,0.68], \omega_{3}=[0.47,0.48], \omega_{4}=[0.16,0.82]$ and $\omega_{5}=[0.35,0.63]$.

Table 1: Picture fuzzy decision-making matrix

\begin{tabular}{|l|l|l|l|l|l|}
\hline & C1 & C2 & C3 & C4 & C5 \\
\hline X1 & {$[0.78,0.11,0.11]$} & {$[0.85,0.05,0.10]$} & {$[0.80,0.09,0.11]$} & {$[0.64,0.27,0.08]$} & {$[0.63,0.29,0.08]$} \\
\hline X2 & {$[1.00,0.00,0.00]$} & {$[1.00,0.00,0.00]$} & {$[0.85,0.05,0.10]$} & {$[1.00,0.00,0.00]$} & {$[1.00,0.00,0.00]$} \\
\hline X3 & {$[0.76,0.12,0.11]$} & {$[0.70,0.20,0.10]$} & {$[0.58,0.34,0.08]$} & {$[0.43,0.53,0.04]$} & {$[0.37,0.52,0.11]$} \\
\hline X4 & {$[1.00,0.00,0.00]$} & {$[1.00,0.00,0.00]$} & {$[0.82,0.07,0.11]$} & {$[1.00,0.00,0.00]$} & {$[1.00,0.00,0.00]$} \\
\hline
\end{tabular}

Step 2. Considering that cost is a criterion of cost type, it must transform cost type criterion into that of benefit type. With Eq. (12), the normalized picture fuzzy decision matrix is shown in Tab.2. 
Table 2: Normalized Picture fuzzy decision-making matrix

\begin{tabular}{|l|l|l|l|l|l|}
\hline & $\mathrm{C} 1$ & $\mathrm{C} 2$ & $\mathrm{C} 3$ & $\mathrm{C} 4$ & $\mathrm{C} 5$ \\
\hline $\mathrm{X} 1$ & {$[0.78,0.11,0.11]$} & {$[0.05,0.85,0.10]$} & {$[0.80,0.09,0.11]$} & {$[0.64,0.27,0.08]$} & {$[0.63,0.29,0.08]$} \\
\hline $\mathrm{X} 2$ & {$[1.00,0.00,0.00]$} & {$[0.00,1.00,0.00]$} & {$[0.85,0.05,0.10]$} & {$[1.00,0.00,0.00]$} & {$[1.00,0.00,0.00]$} \\
\hline $\mathrm{X} 3$ & {$[0.76,0.12,0.11]$} & {$[0.20,0.70,0.10]$} & {$[0.58,0.34,0.08]$} & {$[0.43,0.53,0.04]$} & {$[0.37,0.52,0.11]$} \\
\hline $\mathrm{X} 4$ & {$[1.00,0.00,0.00]$} & {$[0.00,1.00,0.00]$} & {$[0.82,0.07,0.11]$} & {$[1.00,0.00,0.00]$} & {$[1.00,0.00,0.00]$} \\
\hline
\end{tabular}

Step 3. In this paper, PFPIS is defined as $[(1,0,0),(1,0,0),(1,0,0),(1,0,0)$ and $(1,0,0)]$, and PFNIS is defined as $[(0,1,0),(0,1,0),(0,1,0),(0,1,0)$ and $(0,1,0)]$, respectively. And then, with Eq. (5), let $\mathrm{t}=2$ and $\mathrm{p}=2$, the biparametric distance of each green supplier from PFPIS and PFNIS is given in Tab. 3.

Table 3: Distance matric between alternatives and PFPIS and PFNIS

\begin{tabular}{|c|c|c|c|c|c|c|c|c|}
\hline & \multicolumn{3}{|c|}{$d^{+}$} & \multicolumn{4}{|c|}{$d^{-}$} \\
\hline c1 & 0.156 & 0.816 & 0.167 & 0.816 & 0.686 & 0.000 & 0.674 & 0.000 \\
\hline c2 & 0.108 & 0.816 & 0.216 & 0.816 & 0.738 & 0.000 & 0.616 & 0.000 \\
\hline c3 & 0.142 & 0.738 & 0.315 & 0.128 & 0.702 & 0.108 & 0.509 & 0.719 \\
\hline c4 & 0.264 & 0.816 & 0.450 & 0.816 & 0.562 & 0.000 & 0.369 & 0.000 \\
\hline c5 & 0.275 & 0.816 & 0.476 & 0.816 & 0.550 & 0.000 & 0.356 & 0.000 \\
\hline
\end{tabular}

Step 4. According to Eqs. (8) and (9), four pairs of fractional programming models for alternatives $\mathrm{X}_{i}(i=1,2,3,4)$ can be obtained. Let us illustrate this step by using $\left(C_{1}^{l}, C_{1}^{u}\right)$ as an example.

$$
\begin{array}{r}
C_{i}^{l}=\min \left(\frac{\sqrt{\left(0.156 \omega_{1}\right)^{2}+\left(0.108 \omega_{2}\right)^{2}+\left(0.142 \omega_{3}\right)^{2}+\left(0.264 \omega_{4}\right)^{2}+\left(0.275 \omega_{5}\right)^{2}}}{\left(\begin{array}{c}
\sqrt{\left(0.156 \omega_{1}\right)^{2}+\left(0.108 \omega_{2}\right)^{2}+\left(0.142 \omega_{3}\right)^{2}+\left(0.264 \omega_{4}\right)^{2}+\left(0.275 \omega_{5}\right)^{2}}+ \\
\sqrt{\left(0.686 \omega_{1}\right)^{2}+\left(0.738 \omega_{2}\right)^{2}+\left(0.702 \omega_{3}\right)^{2}+\left(0.562 \omega_{4}\right)^{2}+\left(0.550 \omega_{5}\right)^{2}}
\end{array}\right)}\right) \\
\text { s.t. }\left\{\begin{array}{c}
0.15 \leq \omega_{1} \leq 0.83 ; 0.26 \leq \omega_{2} \leq 0.68 ; 0.47 \leq \omega_{3} \leq 0.48 ; \\
0.16 \leq \omega_{4} \leq 0.82 ; 0.35 \leq \omega_{5} \leq 0.63
\end{array}\right.
\end{array}
$$

, and

$$
\begin{gathered}
C_{i}^{u}=\max \left(\frac{\sqrt{\left(0.156 \omega_{1}\right)^{2}+\left(0.108 \omega_{2}\right)^{2}+\left(0.142 \omega_{3}\right)^{2}+\left(0.264 \omega_{4}\right)^{2}+\left(0.275 \omega_{5}\right)^{2}}}{\left(\begin{array}{c}
\sqrt{\left(0.156 \omega_{1}\right)^{2}+\left(0.108 \omega_{2}\right)^{2}+\left(0.142 \omega_{3}\right)^{2}+\left(0.264 \omega_{4}\right)^{2}+\left(0.275 \omega_{5}\right)^{2}}+ \\
\sqrt{\left(0.686 \omega_{1}\right)^{2}+\left(0.738 \omega_{2}\right)^{2}+\left(0.702 \omega_{3}\right)^{2}+\left(0.562 \omega_{4}\right)^{2}+\left(0.550 \omega_{5}\right)^{2}}
\end{array}\right)}\right) \\
\text { s.t. }\left\{\begin{array}{c}
0.15 \leq \omega_{1} \leq 0.83 ; \\
0.26 \leq \omega_{2} \leq 0.68 ; 0.47 \leq \omega_{3} \leq 0.48 ; \\
0.16 \leq \omega_{4} \leq 0.82 ; 0.35 \leq \omega_{5} \leq 0.63
\end{array}\right.
\end{gathered}
$$

Using WINQSB, the optimal values of the objective function given in these models are $C_{1}^{l}=$ 0.0213 and $C_{1}^{u}=0.0829$, i.e., the relative-closeness coefficient interval of green supplier $\mathrm{X} 1$ is $\mathrm{R} 1=$ $[0.0213,0.0829]$. Similarly, the relative-closeness intervals of the green suppliers $\mathrm{X}(\mathrm{k})(\mathrm{k}=2,3,4)$ are respectively obtained as $\mathrm{R} 2=[0.9908,0.992], \mathrm{R} 3=[0.079,0.3165]$ and $\mathrm{R} 4=[0.5771,0.9747]$.

Step 5. Based on those relative closeness intervals of the green suppliers, with Eq. (10), the likelihood probability values of the pairwise components of $\mathrm{X}(\mathrm{k})(\mathrm{k}=1,2,3,4)$ can be calculated as follows:

$$
P=P\left(p_{s t}\right)=\left[\begin{array}{cccc}
0.50 & 0.00 & 0.01 & 0.00 \\
1.00 & 0.50 & 1.00 & 1.00 \\
0.99 & 0.00 & 0.50 & 0.54 \\
0.576 & 0.00 & 0.00 & 0.50
\end{array}\right]
$$

Step 6: The optimal degrees of the membership for the green suppliers $\mathrm{X}_{k}(k=1,2,3,4)$ can be obtained as rank $(\mathrm{X} 1)=0.126, \operatorname{rank}(\mathrm{X} 2)=0.375, \operatorname{rank}(\mathrm{X} 3)=0.207$ and $\operatorname{rank}(\mathrm{X} 4)=0.292$, respectively. This shows that $\operatorname{rank}(\mathrm{X} 1)<\operatorname{rank}(\mathrm{X} 3)<\operatorname{rank}(\mathrm{X} 4)<\operatorname{rank}(\mathrm{X} 2)$, and hence X2 is the best green supplier. This order is the same as the result in [24] and explains the validity of our proposed method. 


\subsection{Comparison with the result of TOPSIS method}

Now, we make a comparative analysis of the proposed method with the existing TOPSIS approach. First, by extending intuitionistic fuzzy weighs to picture fuzzy ones, we have the picture fuzz weight vectors of criteria as $w_{1}=[0.15,0.83,0.02], w_{2}=[0.26,0.68,0.05], w_{3}=[0.47,0.48,0.05], w_{4}$ $=[0.16,0.82,0.03]$ and $w_{5}=[0.35,0.63,0.03]$. Considering that picture fuzzy set is a special form of neutrosophic sets, we have the weighted picture fuzzy matrix by using the operating laws of neutrosophic sets defined in [19], which is shown in Tab. 4.

Table 4: Weighted Picture fuzzy decision-making matrix

\begin{tabular}{|l|l|l|l|l|l|}
\hline & C1 & C2 & C3 & C4 & C5 \\
\hline X1 & {$[0.117,0.849,0.002]$} & {$[0.221,0.696,0.005]$} & {$[0.376,0.527,0.006]$} & {$[0.102,0.869,0.002]$} & {$[0.221,0.737,0.002]$} \\
\hline X2 & {$[0.150,0.830,0.000]$} & {$[0.260,0.680,0.000]$} & {$[0.400,0.506,0.005]$} & {$[0.160,0.820,0.000]$} & {$[0.350,0.630,0.000]$} \\
\hline X3 & {$[0.114,0.850,0.002]$} & {$[0.182,0.744,0.005]$} & {$[0.235,0.657,0.004]$} & {$[0.069,0.915,0.001]$} & {$[0.130,0.822,0.003]$} \\
\hline X4 & {$[0.150,0.830,0.000]$} & {$[0.260,0.680,0.000]$} & {$[0.385,0.516,0.006]$} & {$[0.160,0.820,0.000]$} & {$[0.350,0.630,0.000]$} \\
\hline
\end{tabular}

Next, PFPIS is defined as $[(1,0,0),(1,0,0),(1,0,0),(1,0,0)$ and $(1,0,0)]$, and PFNIS is defined as $[(0,1,0),(0,1,0),(0,1,0),(0,1,0)$ and $(0,1,0)]$, respectively. According to Eq. (1), the normalized picture Hamming distance of each green supplier from PFPIS and PFNIS is given in Tab. 5.

Table 5: Distance matric between alternatives and PFPIS and PFNIS

\begin{tabular}{|c|c|c|c|c|c|c|c|c|c|c|}
\hline & \multicolumn{7}{|c|}{$d^{+}$} & & \multicolumn{5}{|c|}{$d^{-}$} & $\mathrm{C}_{3}$ \\
\hline & $\mathrm{C}_{1}$ & $\mathrm{C}_{2}$ & $\mathrm{C}_{3}$ & $\mathrm{C}_{4}$ & $\mathrm{C}_{5}$ & $\mathrm{C}_{1}$ & $\mathrm{C}_{2}$ & $\mathrm{C}_{3}$ & $\mathrm{C}_{4}$ & $\mathrm{C}_{5}$ \\
\hline $\mathrm{X} 2$ & 1.734 & 1.480 & 1.156 & 1.769 & 1.519 & 0.271 & 0.530 & 0.855 & 0.236 & 0.486 \\
\hline $\mathrm{X} 3$ & 1.739 & 1.420 & 1.112 & 1.660 & 1.280 & 0.320 & 0.580 & 0.899 & 0.340 & 0.720 \\
\hline $\mathrm{X} 4$ & 1.680 & 1.420 & 1.426 & 1.848 & 1.696 & 0.266 & 0.443 & 0.582 & 0.155 & 0.310 \\
\hline
\end{tabular}

At last, we use the relative-closeness coefficient of an alternative to determine the final ranking, and we have $\operatorname{rank}(\mathrm{X} 1)=0.237, \operatorname{rank}(\mathrm{X} 2)=0.286, \operatorname{rank}(\mathrm{X} 3)=0.175$ and $\operatorname{rank}(\mathrm{X} 4)=0.283$, respectively. This shows that $\operatorname{rank}(\mathrm{X} 3)<\operatorname{rank}(\mathrm{X} 1)<\operatorname{rank}(\mathrm{X} 4)<\operatorname{rank}(\mathrm{X} 2)$, and hence X2 is the best green supplier. This order is the same as our method proposed in this study.

\section{Conclusion}

PFS is a useful tool to express inherent uncertainty or imprecision of decision makers' cognitive information in human decision making process. In this paper, we have proposed a method for solving the multi-criteria decision making problems under the Picture fuzzy environment, in which the ratings of alternatives on criteria and weights of criteria are represented by PFSs and IFSs, respectively. Moreover, some fractional programming models are established to obtain a relative closeness coefficient interval where preference information is independently determined for each alternative. This allows us to use flexible ways to simulate real decision scenarios. Finally, a numerical analysis is conducted to illustrate the practicality and effectiveness of the proposed MCMD approach. In future research, the proposed method could be extended to interval-valued picture fuzzy sets and trapezoidal picture fuzzy sets. Another interesting direction could be to develop other types of picture fuzzy aggregation operators.

\section{Funding}

This research was funded by The Social Science Foundation of Jiangsu Province (NO. 16GLB014).

\section{Conflict of interest}

The author declare no conflict of interest. 


\section{References}

[1] Abdel-Basset, M.; Manogaran, G.; Gamal, A.; Smarandache, F. (2018). A hybrid approach of neutrosophic sets and DEMATEL method for developing supplier selection criteria, Design Automation for Embedded Systems, 22, 257-278, 2018.

[2] Akcan, S.; Taş, M. A. (2019). Green supplier evaluation with SWARA -TOPSIS integrated method to reduce ecological risk factors, Environmental Monitoring and Assessment, 191: 736, 2019.

[3] Atanassov, K. (1986). Intuitionistic fuzzy sets, Fuzzy Sets and Systems, 20, 87--96, 1986.

[4] Banaeian, N.; Mobli, H.; Fahimnia, B.; Nielsen, I. E. (2018). Green supplier selection using fuzzy group decision making methods, a case study from the agri-food industry, Computers \& Operations Research, 89(1), 337--347, 2018.

[5] Boran, F. E.; Akay, D. (2014). A biparametric similarity measure on intuitionistic fuzzy sets with applications to pattern recognition, Information Sciences, 255, 45—57, 2014.

[6] Celik, E.; Erdogan, M.; Gumus, A. T. (2016). An extended fuzzy TOPSIS-GRA method based on different separation measures for green logistics service provider selection, International Journal of Environmental Science and Technology, 13(5), 1377-1392, 2016.

[7] Cuong, B. C.; Kreinovich, V. (2013). Picture Fuzzy Sets-a new concept for computational intelligence problems, 2013 Third World Congress on Information and Communication Technologies (WICT 2013), 12: 1-6, 2013.

[8] Dzitac, I.; Filip, F.G.; Manolescu, M.J. (2017). Fuzzy Logic Is Not Fuzzy: World-renowned Computer Scientist Lofti A. Zadeh, International Journal of Computers Communications \& Control, 12(6), 748-789, 2017.

[9] Eda, B.; Zahir, I.; Cengiz, K. (2018). Pythagorean fuzzy CODAS and its application to supplier selection in a manufacturing firm, Journal of Enterprise Information Management, 31(4), 550$564,2018$.

[10] Fallahpour, A.; Olugu, E. U.; Musa, S. N.; Dariush, K.; Kuan, Y. W. (2016) An integrated model for green supplier selection under fuzzy environment: application of data envelopment analysis and genetic programming approach, Neural Computing \& Applications, 27(3), 707-725, 2016.

[11] Gao, H. X.; Ju, Y. B.; Ernesto, S. G.; Zhang, W. K. (2020). Green supplier selection in electronics manufacturing: An approach based on consensus decision making, Journal of Cleaner Production, $245,118781,2020$.

[12] Garg, H.; Arora, R. (2018). A nonlinear-programming methodology for multi- attribute decision making problem with interval-valued intuitionistic fuzzy soft sets information, Applied Intelligence, 48, 2031--2046, 2018.

[13] Gitinavard, H.; Ghaderi, H.; Pishvaee, M. S. (2018). Green supplier evaluation in manufacturing systems: a novel interval-valued hesitant fuzzy group outranking approach, Soft Computing, 3, $1-20,2018$.

[14] Govindan, K.; Sivakumar, R. (2016). Green supplier selection and order allocation in a low carbon paper industry: integrated multi-criteria heterogeneous decision-making and multi-objective linear programming approaches, Annals of Operations Research, 238, 243--276, 2016.

[15] Karsak, E. E.; Dursun, M. (2015). An integrated fuzzy MCDM approach for supplier evaluation and selection, Computers \& Industrial Engineering, 82: 82--93, 2015.

[16] Li, J; Wang, J. Q. (2017). An extended QUALIFLEX method under probability hesitant fuzzy environment for selecting green suppliers, International Journal of Fuzzy Systems, 19(6), 1866$1879,2017$. 
[17] Liu, H. C.; Quan, M. Y.; Li, Z. W.; Wang, Z. L. (2019). A new integrated MCDM model for sustainable supplier selection under interval-valued intuitionistic uncertain linguistic environment, Information Sciences, 486, 254-270, 2019.

[18] Liu, P. D.; Gao, H.; Ma, J. H. (2019). Novel green supplier selection method by combining quality function deployment with partitioned Bonferroni mean operator in interval type-2 fuzzy environment, Information Sciences, 490, 292--316, 2019.

[19] Liu, P.; Wang, Y. (2014). Multiple attribute decision making method based on single-valued neutrosophic normalized weighted Bonferroni mean, Neural Computing \& Applications, 25:2001$-2010,2014$.

[20] Lo, H. W.; Liou, J. J. H.; Wang, H. S.; Tsai, Y. S. (2018). An integrated model for solving problems in green supplier selection and order allocation, Journal of Cleaner Production, 190(20), 339-352, 2018 .

[21] Memaria, A.; Dargib, A.; Jokara, M. R. A.; Ahmadc, R.; Rahim, A. R. A. (2019). Sustainable supplier selection, A multi-criteria intuitionistic fuzzy TOPSIS method, Journal of Manufacturing Systems, 50, 9--24, 2019.

[22] Mousakhani, S.; Nazari-Shirkouhi, S.; Bozorgi-Amiri, A. (2017). A novel interval type-2 fuzzy evaluation model based group decision analysis for green supplier selection problems: A case study of battery industry, Journal of Cleaner Production, 168, 205-218, 2017.

[23] Pravin, K.; Rajesh, S. K.; Anurika, V. (2017). Suppliers' green performance evaluation using fuzzy extended ELECTRE approach, Clean Technologies and Environmental Policy, 19, 809--821, 2017.

[24] Rouyendegh, B. D.; Yildizbasi, A.; Üstünyer, P. (2019). Intuitionistic fuzzy TOPSIS method for green supplier selection problem, Soft Computing, DOI:https://doi.org/10.1007/s00500-01904054-8, 2019.

[25] Shen, L. X.; Olfat, L.; Govindan, K.; Khodaverdi, R.; Diabat, A. (2013). A fuzzy multi criteria approach for evaluating green supplier's performance in green supply chain with linguistic preferences, Resources, Conservation \& Recycling, 74, 170-179, 2013.

[26] Son, L. H. (2015). DPFCM, A novel distributed picture fuzzy clustering method on picture fuzzy sets, Expert Systems with Applications, 42, 51-66, 2015.

[27] Wang, L.; Zhang, H. Y.; Wang, J. Q.; Li, L. (2017). Picture fuzzy normalized projection based VIKOR method for the risk evaluation of construction project, Applied Soft Computing, 64, 216$-226,2017$.

[28] Wu, J.; Liu, Y. J. (2013). An approach for multiple attribute group decision making problems with interval-valued intuitionistic trapezoidal fuzzy numbers, Computers \& Industrial Engineering, 66, 311--324, 2013.

[29] Wu, Q.; Zhou, L.; Chen, Y.; Chen, H. Y. (2019). An integrated approach to green supplier selection based on the interval type-2 fuzzy best-worst and extended VIKOR methods, Information Sciences, 502, 394-417, 2019.

[30] Xu, Z. S. (2011). A ranking arithmetic for fuzzy mutual complementary judgment matrices, Journal of Systems Engineering, 16 (4), 311-314, 2001.

[31] Xu, Z. S.; Da, Q. L. (2003). A likelihood based method for priorities of interval judgment matrices, Chinese Journal of Management Science, 11, 63--65, 2003.

[32] Zeng, S. Z.; Chen, S. M.; Wei, K. L. (2019). Multi-attribute decision making based on novel score function of intuitionistic fuzzy values and modified VIKOR method, Information Sciences, 488, 76--92, 2019. 


\section{(c) (i) (8)}

Copyright (C)2020 by the authors. Licensee Agora University, Oradea, Romania.

This is an open access article distributed under the terms and conditions of the Creative Commons Attribution-NonCommercial 4.0 International License.

Journal's webpage: http://univagora.ro/jour/index.php/ijccc/

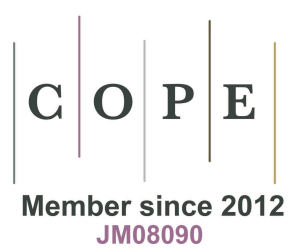

This journal is a member of, and subscribes to the principles of, the Committee on Publication Ethics (COPE).

https://publicationethics.org/members/international-journal-computers-communications-and-control

Cite this paper as:

Cao, G.(2020). A Multi-criteria Picture Fuzzy Decision-making Model for Green Supplier Selection based on Fractional Programming, International Journal of Computers Communications \& Control, 15(1), 1002, 2020. https://doi.org/10.15837/ijccc.2020.1.3762 